\title{
Microscopic Polyangiitis in Systemic Sclerosis
}

\author{
Hiroshi Hashimoto ${ }^{1,2}$ \\ ${ }^{1}$ Juntendo University School of Medicine, 2-1-1 Hongo, Bunkyoku, Tokyo 113-0033, Japan \\ ${ }^{2}$ Division of Rheumatology, Aiwakai Medical Corporation, 1-22-23 Kamiyouga, Setagayaku, Tokyo 158-0098, Japan
}

Correspondence should be addressed to Hiroshi Hashimoto, hashi@med.juntendo.ac.jp

Received 14 May 2010; Accepted 12 July 2010

Academic Editor: Eswar Krishnan

Copyright () 2010 Hiroshi Hashimoto. This is an open access article distributed under the Creative Commons Attribution License, which permits unrestricted use, distribution, and reproduction in any medium, provided the original work is properly cited.

\begin{abstract}
AAV in SSc is described from the point of view of MPA. Some of reported SSc cases with AAV are thought to exhibit the characteristic clinical manifestations of MPA, although ANCA positivity in SSc is uncommon. MPA is clinically characterized by a multisystemic disease such as RPGN, pulmonary hemorrhage, mononeuritis, and skin involvement, as well as other manifestations in conjunction with high levels of inflammatory activity such as high ESR or CRP. It is also characterized by a high frequency of MPO-ANCA, showing predominant pANCA by IIF. When rapid renal failure or RPGN with active urine sediments, pulmonary hemorrhage and/or systemic inflammatory manifestations are observed in patients with SSc having positive ANCA, the possibility of MPA should always be considered. If SSc patients with MPA have life-threatening visceral involvement such as the above clinical manifestations, the patients should be treated with induction therapy using cyclophosphamide, methotrexate, corticosteroids, or plasmapheresis, etc. according to the severity of the disease soon after the diagnosis of MPA. It is important not to overlook characteristic clinical manifestations of AAV during the course of the disease in SSc in order to diagnose MPA early.
\end{abstract}

\section{Introduction}

Systemic sclerosis (SSc), which mostly affects middle-aged women, is a chronic disorder of connective tissue characterized by inflammation and fibrosis and by degenerative changes in the blood vessels, skin, synovium, skeletal muscle, and certain internal organs, notably the gastrointestinal tract, lung, heart, and kidney [1]. The life-threatening visceral involvements in SSc include scleroderma renal crisis, pulmonary hypertension, and lung fibrosis.

On the other hand, microscopic polyangiitis (MPA), which mostly affects elderly people, is a systemic disease characterized by vasculitis involving small blood vessels, particularly the glomerular and pulmonary capillaries, and serologically by antineutrophil cytoplasmic antibody (ANCA) positivity [2-4]. MPA is also well known to present as one form of primary ANCA-associated vasculitis (AAV) as well as Wegener's granulomatosis (WG) [2]. Clinical manifestations in this disease include rapidly progressive glomerulonephritis (RPGN) and pulmonary hemorrhage, which are life-threatening visceral involvements.

Cases of SSc patients with AAV have been reported, although ANCA positivity in SSc is uncommon. Some of these cases are thought to exhibit the characteristic clinical manifestations of MPA. In this paper, AAV in SSc will be described from the point of view of MPA.

\section{ANCA and MPA}

ANCA is well known to be associated with small-sized vasculitic disorders such as MPA, WG, and allergic granulomatous angiitis (AGA) in primary systemic vasculitides. These diseases are also known as primary ANCA-associated vasculitides. There are two major distinct subtypes of ANCA. One subtype is an antibody against myeloperoxidase (MPO), which stains in a perinuclear pattern (pANCA) by indirect immunofluorescence (IIF) using a neutrophil substrate, and the other subtype is an antibody against proteinase 3 (PR3), which stains in a diffuse granular cytoplasmic pattern (cANCA) by IIF. Enzyme-linked immunosorbent assay (ELISA) is used for target-specific ANCA determination [5]. MPO-ANCA can be found in MPA and AGA more frequently than in WG. On the other hand, PR3-ANCA is more specific in WG than the other primary systemic vasculitides. The association of MPA with MPO-ANCA is reported to be in 
TABLe 1: Comparison of clinical manifestations in MPA between French and Japanese studies.

\begin{tabular}{|c|c|c|}
\hline & French study [4] & Japanese study [19] \\
\hline Cases & 85 & 63 \\
\hline Age, mean (range) years & $56.8(16-86)$ & $59.0(13-91)$ \\
\hline Sex ratio, Male: Female & 47 versus 38 & 19 versus 44 \\
\hline Fever & $55.30 \%$ & $71.00 \%$ \\
\hline Weight loss & $72.90 \%$ & $42.90 \%$ \\
\hline Skin involvement & $62.40 \%$ & $24.20 \%$ \\
\hline Arthralgias & $50.60 \%$ & $62.90 \%$ \\
\hline Myalgias & $48.20 \%$ & $50.80 \%$ \\
\hline Renal involvement & $78.80 \%$ & $87.30 \%$ \\
\hline Renal insufficiency & (47/67) 70.1\% & $49.20 \%$ \\
\hline Rapidly progressive GN & - & $66.70 \%$ \\
\hline Proteinuria & (54/67) 80.6\% & $93.10 \%$ \\
\hline Hematuria & (45/67) $67.2 \%$ & $76.70 \%$ \\
\hline Lung involvement & $24.70 \%$ & $63.50 \%$ \\
\hline Alveolar hemorrhage & $11.80 \%$ & $22.20 \%$ \\
\hline Pneumonitis & $10.60 \%$ & $33.30 \%$ \\
\hline Pleiritis & $5.90 \%$ & $19.00 \%$ \\
\hline Mononeuritis multiplex & $57.60 \%$ & $30.00 \%$ \\
\hline Central nervous system involvement & $11.80 \%$ & $6.30 \%$ \\
\hline Gastrointestinal tract involvement & $30.60 \%$ & $6.30 \%$ \\
\hline Hypertension & $34.10 \%$ & $41.30 \%$ \\
\hline Myocardial infarction & $2.40 \%$ & $1.60 \%$ \\
\hline Pericariditis & $10.60 \%$ & $3.20 \%$ \\
\hline Cardiac failure & $17.60 \%$ & $3.20 \%$ \\
\hline
\end{tabular}

the range of $40-80 \%$, and MPA with MPO-ANCA is noted to be frequently associated with necrotizing glomerulonephritis and/or pulmonary capillaritis, namely, a pulmonary-renal syndrome similar to that observed in Goodpasture syndrome or WG $[3,4]$. Furthermore, renal-limited vasculitis, which is characterized by pauci-immune focal necrotizing crescentic glomerulonephritis (pFNCGN), is classified as MPA using European Medicines Agency (EMEA) algorithm [6]. Interestingly, MPO-ANCA-associated vasculitides are more common in Asian countries than in the USA or Europe, where $80 \%$ of AAV is PR-3 ANCA [6, 7] and ANCA-positive, while anti-MPO-negative patients with MPA most often have antibody specificity for PR3 [3].

In Japan, MPO-ANCA has been reported to account for $90 \%$ of cases of AAV $[8,9]$. Therefore, renal-limited AAV in Japan is almost exclusively MPA, whereas in the UK only $41 \%$ of cases of renal limited AAV have MPA, although the overall incidences of renal vasculitis are similar in the UK and Japan [6].

On the other hand, ANCA has also been described in patients with rheumatic autoimmune diseases, including rheumatoid arthritis, systemic lupus erythematosus, Sjögren's syndrome, polymyositis/dermatomyositis, and antiphospholipid syndrome. The fluorescence patterns encountered in these diseases are mostly pANCA. A multitude of target antigens, including not only MPO but also lactoferrin, elastase, lysozyme, cathepsin G, and bactericidal/permeability-increasing protein (BPI) have been described in rheumatic autoimmune diseases $[3,10]$. However, ANCA in SSc is uncommon, showing a positive rate ranging from 0 to $18 \%$ according to investigators $[3,11-17]$, in spite of the development of many autoantibodies, such as antitopoisomerase-I (Scl-70) antibodies, anticentromere antibodies, and rheumatoid factors, in SSc. ANCA in SSc usually shows pANCA and/or anti-MPO-ANCA, although $\mathrm{BPI}$ and cathepsin $\mathrm{G}$ were reported to be the major antigenic targets of ANCA seen in patients with SSc [18].

\section{Characteristics of MPA}

MPA is clinically characterized by a multisystemic disease such as RPGN, alveolar hemorrhage, mononeuritis, and skin involvement, as well as other manifestations. It is also characterized by a high frequency of MPO-ANCA, showing predominant pANCA by IIF as mentioned above. Pathologically, inflammation and necrosis in various sites of vessels including arteries, arterioles, capillaries, and venules are observed, and pFNCGN characteristically occurs in the context of MPA as well as WG. The severity of the injury requires immediate treatment with immunosuppressive drugs including cyclophosphamide and corticosteroids.

There are also some differences in the clinical manifestations of MPA, in addition to the clinical phenotype of renal AVV, between Europe and Japan. Table 1 shows 
TAble 2: Proposed diagnostic criteria for microscopic polyangiitis (MPA). (Research Committee on Intractable Vasculitis and Research Committee on Epidemiology of Intractable Diseases, The Ministry of Health and Welfare of Japan, 1998).

(1) Clinical manifestations

(i) rapidly progressive glomerulonephritis

(ii) pulmonary hemorrhage or interstitial pneumonia

(iii) organ involvement besides kidney and lung:

purpura, subcutaneous bleeding, gastrointestinal bleeding, mononeuritis multiplex, and so forth

(2)Histological findings

necrotizing vasculitis in capillaries, venules, or arterioles, with perivascular inflammatory infiltrate

(3) Laboratory findings

(i) positive MPO-ANCA

(ii) elevated level of CRP

(iii) proteinuria and/or hematuria, or elevated levels of BUN and/or creatinine

(iv) chest X-ray findings: infiltration (pulmonary hemorrhage), and/or interstitial pneumonitis

(4) Diagnosis

(i) Definite

(a) At least two clinical manifestations with the histological findings

(b) At least two clinical manifestations including items (i) or (ii), and positive MPO-ANCA

(ii) Probable

(a) At least three clinical manifestations

(b) One clinical manifestation and positive MPO-ANCA

(5) Exclusion diseases

(i) polyarteritis nodosa

(ii) Wegener's granulomatosis

(iii) allergic granulomatous angiits (Churg-Strauss syndrome)

(iv) Goodpasture syndrome

a comparison of clinical findings of MPA in the French Vasculitis Study Group reported by Guillevin et al. [4] and the Japanese Nationwide Epidemiological Survey [19] reported by Hashimoto et al. The observed differences might be expected because the same diagnostic criteria were not used, although the inclusion criteria for the diagnosis of MPA were almost the same. Table 2 shows the diagnostic criteria of MPA used in the Japanese Nationwide Epidemiological Survey. Interestingly, the female: male sex ratio was 1.23 in the French study, which contrasts with 0.43 in the Japanese study, although the mean age at diagnosis was almost the same. The incidence of clinical manifestations including fever and weight loss in the French study was greater than that in the Japanese study, but the incidences of arthralgias and myalgias were almost the same. Concerning visceral involvement, the incidence of renal involvement and lung involvement, such as alveolar hemorrhage, pneumonitis, and pleuritis, in the Japanese study was greater than that in the French study; in contrast, the incidence of mononeuritis multiplex, central nervous system involvement, and gastrointestinal tract involvement in the French study was greater than that in the Japanese study. The frequencies of hypertension were $34.1 \%$ in the French study and $41.3 \%$ in the Japanese study.

In the French study, ANCA was present in $74.5 \%$ of patients, of whom $86.8 \%$ had pANCA and the remainder had cANCA. In the Japanese study, pANCA was present in $90.4 \%$ of patients and cANCA was present in $9.6 \%$. Among pathological findings in the Japanese study, necrotizing vasculitis was present in 32 out of 55 patients and crescentic glomerulonephritis was present in 41 out of 47 patients (not indicated in the French study). In the Japanese study, most of the patients were treated with corticosteroids (93.4\%) and immunosuppressant drugs (71.0\%). The mortality rate was $12.9 \%$ and the most frequent cause of death was infection (36.5\%) followed by alveolar hemorrhage (17.3) and renal failure (13.5\%). Guillevin et al. [4] noted that in the French study, deaths were less frequent when patients had been treated with corticosteroids and immunosuppressive drugs, but relapse of MPA was common.

\section{AAV in SSc}

SSc is divided into two major clinical subsets, namely diffuse cutaneous and limited cutaneous disease, which are distinguished from one another primarily by the degree and extent of skin involvement [20]. Overlap syndrome, which has either diffuse or limited cutaneous disease and typical features of one or more of other connective tissue diseases, is also present [1]. Since the identification of antitopoisomerase-I (anti-Scl-70 antibody) and anticentromere antibodies, it has been well known that antitopoisomerase-1 antibodies are associated with diffuse cutaneous disease as well as evident renal involvement and pulmonary interstitial fibrosis, and anticentromere antibodies are associated with limited cutaneous disease as 
well as pulmonary hypertension. Anti-U1RNP and anti-Ku antibodies are observed in patients with overlap syndrome of SSc and myositis. Although the number of cases with overlap syndrome of SSc and MPA among the cases of SSc with AAV that have been reported is unknown, several cases of overlap syndrome were suggested to be present by Rho et al. [21] who analyzed and reviewed the clinical characteristics of SSc with AAV in 50 cases reported in 31 articles. In this study, MPO-ANCA was the predominant type (72\%), but PR-3 ANCA was also found in some cases (24\%). The authors noted that 33 out of 50 cases had definite features of AAV with pathological or clinical evidence including crescentic glomerulonephritis, RPGN, pulmonary hemorrhage, skin and/or nerve vasculitis, and necrotizing vasculitis of muscle. High levels of inflammatory activity, such as high ESR or CRP, or abnormal urinary sediments, were also indicated. Cases with MPO-ANCA had a higher prevalence of renal impairment and pulmonary hemorrhage than those with PR-3 ANCA. Having antitopoisomerase1 antibodies made the development of AAV in SSc three times more likely than that in patients who had neither antibody. The mortality rate was $39.4 \%$ with virtually all of the deaths occurring within 1 year. The major causes of deaths included infection or septic shock, pulmonary hemorrhage, intracerebral hemorrhage due to coagulopathy, and acute cardiac failure. These findings strongly suggest the coexistence of MPA and strongly contrast with the survival rates and causes of deaths that are generally recorded for patients with SSc.

Although the association of normotensive rapid renal failure and MPO-ANCA that suggested the existence of a renal specific subset apart from scleroderma renal crisis was noted [13], nearly one-third of the MPA patients with renal involvement were hypertensive $[4,19]$, and hypertension was not an essential concomitant of renal failure due to SSc even though renal arterial stenosis was observed pathologically in SSc [22]. Blood pressure measurement cannot indicate whether or not scleroderma renal crisis or RPGN associated with AAV exists. However, the association of normotensive renal failure with microangiopathic hemolytic anemia in SSc was indicated [23]. When rapid renal failure or RPGN with active urine sediments or systemic inflammatory manifestations is observed in patients with SSc, the possibility of MPA should always be considered. In these cases, a renal biopsy should be considered to evaluate histological findings because the use of basic laboratory indicators, such as hematuria, proteinuria, or serum creatinine level, is considerably limited in facilitating the prediction of the site affected by vasculitis $[2,4,24]$. The characteristic renal histopathological findings of MPA are pauci-immune necrotizing and/or crescentic focal necrotizing glomerulonephritis as well as small vessel arteritis, which is identical to the lesions seen in WG or renal-limited vasculitis, which is a subtype of MPA [6, 9]. However, this finding distinguishes the disease from polyarteritis nodosa and SSc with fulminating course of malignant hypertension in which mucoid thickening of proximal interlobular arteries and fibrinoid necrosis in distal interlobular arterioles are specific for SSc [25].
TABLE 3: Differences in vascular clinical findings between SSc and MPA.

\begin{tabular}{|c|c|c|}
\hline & SSc & MPA \\
\hline $\begin{array}{l}\text { Raynaud's } \\
\text { phenomenon }\end{array}$ & + & - \\
\hline digital ischemia & + & - \\
\hline skin ulcers & + & + \\
\hline skin nodules & - & + \\
\hline Purpura & - & + \\
\hline $\begin{array}{l}\text { nail bed } \\
\text { change/telangiectasia }\end{array}$ & + & - \\
\hline Myositis & + & + \\
\hline interstitial pneumonia & + & + \\
\hline honeycomb lung & + & - \\
\hline alveolar hemorrhage & - & + \\
\hline $\begin{array}{l}\text { pulmonary } \\
\text { hypertension }\end{array}$ & + & - \\
\hline RPGN & - & + \\
\hline $\begin{array}{l}\text { scleroderma renal } \\
\text { crisis }\end{array}$ & + & - \\
\hline $\begin{array}{l}\text { mononeuritis } \\
\text { multiplex }\end{array}$ & - & + \\
\hline CNS vasculitis & - & + \\
\hline \multicolumn{3}{|l|}{ Histological findings; } \\
\hline fibrinoid necrosis & arteries, arterioles & capillaries, arterioles \\
\hline intimal hyperplasia & + & - \\
\hline capillaritis & - & + \\
\hline pFNCGN & - & + \\
\hline $\begin{array}{l}\text { SSc: scleroderma } \\
\text { MPA: microscopic polyang } \\
\text { RPGN: rapidly progressive } \\
\text { CNS: central nervous syster } \\
\text { pFNCGN: pauci-immune f }\end{array}$ & $\begin{array}{l}\text { iitis } \\
\text { glomerulonephritis } \\
\text { m } \\
\text { focal necrotizing cres }\end{array}$ & 1 \\
\hline
\end{tabular}

Mortality is greatest in the setting of pulmonary-renal syndrome in MPA. Pulmonary lesion is a small-vessel vasculitis, with fibrinoid necrosis of capillaries, leading to alveolar septal disruption, blood-filled alveoli, and the clinical sequelae of dyspnea, cough, and/or hemoptysis. The relative risk of death in MPA has been calculated to be 8 times higher in patients with pulmonary hemorrhage [26]. On the other hand, pulmonary hypertension, which is mostly observed in limited cutaneous scleroderma, is rare in MPA, although a few cases with WG and MPA accompanied with pulmonary hypertension have been reported [27]. In SSc, interstitial fibrosis was found to be the most common pulmonary lesion, showing bilateral fibrosis of lower lung or honeycomb lung in chest X-ray. Its presence correlated well with clinical measurements of restrictive lung disease and decreased diffusing capacity. Arteriolar thickening, described as medial hypertrophy or concentric intimal proliferation, was the most specific lesion in lungs, being noted in $29 \%$ of SSc patients in autopsy cases [22]. The differences in vascular clinical findings between SSc and MPA are shown in Table 3. 
No treatment for SSc is proven to be effective in preventing progression of disease, reversing fibrosis, or improving long-term outcome, although a number of novel agents including anti-interleukin-6, transforming growth factor- $\beta$ directed therapies, and other novel biological agents are being developed [28]. However, if SSc patients with MPA have life-threatening visceral involvement such as rapid renal failure or RPGN and pulmonary hemorrhage, the patients should be treated with induction therapy using cyclophosphamide, methotrexate, corticosteroids, or plasmapheresis, and so forth, according to disease severity soon after the diagnosis of MPA, although attention should be paid to reducing treatment toxicity [29].

\section{Significance of MPO-ANCA in Overlap Syndrome of MPA and SSc}

The etiology of MPA is unknown, but is generally considered to be the result of an interaction between triggering agents and disease susceptibility genes. In primary AAV, MPOANCA as well as PR3-ANCA has been established as a marker for diagnosis and has been implicated in the pathogenesis of vasculitis $[2,30]$. In most patients with MPA and/or SSc with AAV, high titers of MPO-ANCA are associated with disease activity, rises in MPO-ANCA titers precede relapses, and even a case of MPO-ANCA seroconversion associated with fulminant vasculitis in antitopoisomerase1 antibody positive SSc has been reported [31], but MPOANCA titers do not necessarily correlate with disease activity or vasculitis syndrome. There are also the cases without clinical manifestations related to AAV in spite of MPOANCA positivity. The same can be seen for other autoantibodies, such as anti-U1-RNP antibodies, antiphospholipid antibodies, and rheumatoid factor, in rheumatic diseases. Although the reason for cases without clinical manifestations showing MPO-ANCA positivity is not known, the following reasons may be considered: (1) it is a predictive marker for the development of AAV in the future, (2) it is an epiphenomenon, and (3) false positivity. The pathogenic potential of MPO-ANCA to small-vessel vasculitis may be due to not only quantities but also qualities of MPOANCA such as immunoglobulin phenotypes, affinity and/or avidity of MPO-ANCA, and specific risk epitope for MPO-ANCA.

Concerning the source of antigens, attention has been focused on neutrophil extracellular traps (NETs) [32], which are chromatin fibers and are released by ANCA-stimulated neutrophils and contain the targeted autoantigens of PR3 and MPO [33] as well as topoisomerase-1. This may be circumstantial evidence that antitopoisomerase-1 antibodies occur more frequently in SSc with AAV than are usually found in SSc as described in the paper of Rho et al. [21].

Some investigators pointed out that ANCA positivity in SSc is a red flag and draws attention $[17,21]$. This is true, and it is important not to overlook characteristic clinical manifestations of AAV during the course of the disease in SSc.

\section{Abbreviations}

$\begin{array}{ll}\text { AAV: } & \text { ANCA associated vasculitis } \\ \text { AGA: } & \text { Allergic granulomatosis angiitis } \\ \text { ANCA: } & \text { Anti-neutrophil cytoplasmic antibody } \\ \text { BPI: } & \text { Bactericidal/permeability-increasing protein } \\ \text { cANCA: } & \text { Cytoplasmic ANCA } \\ \text { ELISA: } & \text { Enzyme-linked immunosorbent assay } \\ \text { EMEA: } & \text { European Medicines Agency } \\ \text { IIF: } & \text { Indirect immunofluorescence } \\ \text { MPA: } & \text { Microscopic polyangiitis } \\ \text { MPO: } & \text { Myeloperoxidase } \\ \text { NETs: } & \text { Neutrophil extracellular traps } \\ \text { pANCA: } & \text { Perinuclear ANCA } \\ \text { pFNCGN: } & \text { Pauci-immune focal necrotizing crescentic } \\ & \text { glomerulonephritis } \\ \text { RPGN: } & \text { Rapidly progressive glomerulonephritis } \\ \text { SSc: } & \text { Systemic sclerosis } \\ \text { WG: } & \text { Wegener's granulomatosis. }\end{array}$

\section{References}

[1] T. A. Medsger, "Systemic sclerosis (scleroderma): clinical aspect," in Arthritis and Allied Conditions, W. J. Koopman, Ed., pp. 1590-1624, Lippincott Williams \& Wilkins, Philadelphia, Pa, USA, 2001.

[2] J. C. Jennette, R. J. Falk, K. Andrassy et al., "Nomenclature of systemic vasculitides: proposal of an international consensus conference," Arthritis and Rheumatism, vol. 37, no. 2, pp. 187$192,1994$.

[3] G. S. Hoffman and U. Specks, "Antineutrophil cytoplasmic antibodies," Arthritis and Rheumatism, vol. 41, no. 9, pp. 15211537, 1998.

[4] L. Guillevin, B. Durand-Gasselin, R. Cevallos et al., "Microscopic polyangiitis: clinical and laboratory findings in eightyfive patients," Arthritis and Rheumatism, vol. 42, no. 3, pp. 421-430, 1999.

[5] E. C. Hagen, M. R. Daha, J. Hermans et al., "Diagnostic value of standardized assays for anti-neutrophil cytoplasmic antibodies in idiopathic systemic vasculitis," Kidney International, vol. 53, no. 3, pp. 747-753, 1998.

[6] R. A. Watts, D. G. I. Scott, D. R. W. Jayne et al., "Renal vasculitis in Japan and the UK-are there differences in epidemiology and clinical phenotype?" Nephrology Dialysis Transplantation, vol. 23, no. 12, pp. 3928-3931, 2008.

[7] M. Chen, F. Yu, Y. Zhang, W.-Z. Zou, M.-H. Zhao, and H.Y. Wang, "Characteristics of Chinese patients with Wegener's granulomatosis with anti-myeloperoxidase autoantibodies," Kidney International, vol. 68, no. 5, pp. 2225-2229, 2005.

[8] M. Yoshida, T. Iwahori, I. Nakabayashi, M. Akashi, T. Watanabe, and N. Yoshikawa, "In vitro production of myeloperoxidase anti-neutrophil cytoplasmic antibody and establishment ot Th1-type T cell lines from peripheral blood lymphocytes of patients," Clinical and Experimental Rheumatology, vol. 23, no. 2, pp. 227-230, 2005.

[9] S. Fujimoto, S. Uezono, S. Hisanaga et al., "Incidence of ANCA-associated primary renal vasculitis in the Miyazaki prefecture: the first population-based, retrospective, epidemiologic survey in Japan," Clinical Journal of the American Society of Nephrology, vol. 1, no. 5, pp. 1016-1022, 2006. 
[10] B. Malenica, M. Rudolf, and A. Kozmar, "Antineutrophil cytoplasmic antibodies (ANCA): diagnostic utility and potential role in the pathogenesis of vasculitis," Acta Dermatovenerologica Croatica, vol. 12, no. 4, pp. 294-313, 2004.

[11] W. L. Gross, W. H. Schmitt, and C. E. DiplBiol, "Antineutrophil cytoplasmic autoantibody-associated diseases: a rheumatologist's perspective," American Journal of Kidney Diseases, vol. 18, no. 2, pp. 175-179, 1991.

[12] P. A. Merkel, R. P. Polisson, Y. Chang, S. J. Skates, and J. L. Niles, "Prevalence of antineutrophil cytoplasmic antibodies in a large inception cohort of patients with connective tissue disease," Annals of Internal Medicine, vol. 126, no. 11, pp. 866873, 1997.

[13] H. Endo, T. Hosono, and H. Kondo, "Antineutrophil cytoplasmic autoantibodies in 6 patients with renal failure and systemic sclerosis," Journal of Rheumatology, vol. 21, no. 5, pp. 864-870, 1994.

[14] S. Akimoto, O. Ishikawa, T. Tamura, and Y. Miyachi, "Antineutrophil cytoplasmic autoantibodies in patients with systemic sclerosis," British Journal of Dermatology, vol. 134, no. 3, pp. 407-410, 1996.

[15] A. Ruffatti, R. A. Sinico, A. Radice et al., "Autoantibodies to proteinase 3 and myeloperoxidase in systemic sclerosis," Journal of Rheumatology, vol. 29, no. 5, pp. 918-923, 2002.

[16] S. Kiraz, H. Simsek, I. Ertenli, M. Benekli, and A. Kadayifci, "Antineutrophil cytoplasmic antibodies in systemic sclerosis," Clinical Rheumatology, vol. 15, no. 5, pp. 519-520, 1996.

[17] S. Casari, M. Haeney, S. Ferrand, and A. Herrick, "Antineutrophil cytoplasmic antibodies - a "red flag" in patients with systemic sclerosis," Journal of Rheumatology, vol. 29, no. 12, pp. 2666-2667, 2002.

[18] D. Khanna, A. Aggarwal, D. S. Bhakuni, R. Dayal, and R. Misra, "Bactericidal/permeability-increasing protein and cathepsin $\mathrm{G}$ are the major antigenic targets of antineutrophil cytoplasmic autoantibodies in systemic sclerosis," Journal of Rheumatology, vol. 30, no. 6, pp. 1248-1252, 2003.

[19] H. Hashimoto, T. Yano, M. Yoshida, S. Kobayashi, K. Sueishi, N. Tsusaka, et al., "Clinical and epidemiological analysis of anti-neutrophil cytoplasmic antibodies (ANCA) associated vasculitis from a nationwide survey in 1998 in Japan," Annual Report of The Research Committee of Intractable Vasculitis Diseases, The Ministry of Health and Welfare of Japan, 1999.

[20] E. C. LeRoy, C. Black, R. Fleischmajer et al., "Scleroderma (systemic sclerosis): classification, subsets and pathogenesis," Journal of Rheumatology, vol. 15, no. 2, pp. 202-205, 1988.

[21] Y. H. Rho, S. J. Choi, Y. H. Lee, J. D. Ji, and G. G. Song, "Scleroderma associated with ANCA-associated vasculitis," Rheumatology International, vol. 26, no. 5, pp. 465-468, 2006.

[22] W. A. D’Angelo, J. F. Fries, A. T. Masi., and L. E. Shulman, "Pathologic observations in systemic sclerosis (scleroderma). A study of fifty-eight autopsy cases and fifty-eight matched controls," The American Journal of Medicine, vol. 46, no. 3, pp. 428-440, 1969.

[23] D. J. Helfrich, B. Banner, V. D. Steen, and T. A. Medsger Jr., "Normotensive renal failure in systemic sclerosis," Arthritis and Rheumatism, vol. 32, no. 9, pp. 1128-1134, 1989.

[24] K. Joh, E. Muso, H. Shigematsu et al., "Renal pathology of ANCA-related vasculitis: proposal for standardization of pathological diagnosis in Japan," Clinical and Experimental Nephrology, vol. 12, no. 4, pp. 277-291, 2008.

[25] G. P. Rodnan, G. E. Schreiner, and R. L. Black, "Renal involvement in progressive systemic sclerosis (generalized scleroderma)," The American Journal of Medicine, vol. 23, no. 3, pp. 445-462, 1957.
[26] P. A. Bacon and D. Adu, "Microscopic polyangiitis: clinical aspects," in Inflammatory Diseases of Blood Vessels, G. S. Hoffman and C. M. Weyand, Eds., pp. 355-363, Marcel Dekker, New York, NY, USA, 2002.

[27] D. Launay, R. Souza, L. Guillevin et al., "Pulmonary arterial hypertension in ANCA-associated vasculitis," Sarcoidosis Vasculitis and Diffuse Lung Diseases, vol. 23, no. 3, pp. 223-228, 2006.

[28] N. P. Quillinan and C. P. Denton, "Disease-modifying treatment in systemic sclerosis: current status," Current Opinion in Rheumatology, vol. 21, no. 6, pp. 636-641, 2009.

[29] L. Harper, "Recent advances to achieve remission induction in antineutrophil cytoplasmic antibody-associated vasculitis," Current Opinion in Rheumatology, vol. 22, no. 1, pp. 37-42, 2010.

[30] D. J. Schlieben, S. M. Korbet, R. E. Kimura, M. M. Schwartz, and E. J. Lewis, "Pulmonary-renal syndrome in a newborn with placental transmission of ANCAs," American Journal of Kidney Diseases, vol. 45, no. 4, pp. 758-761, 2005.

[31] G. Cheung, G. Chew, R. Wyndham, M. Peters, and S. Riminton, "Myeloperoxidase-antineutrophil cytoplasmic antibody seroconversion and fulminant vasculitis in Scl-70-positive scleroderma," Internal Medicine Journal, vol. 37, no. 3, pp. 205-207, 2007.

[32] V. Brinkmann, U. Reichard, C. Goosmann et al., "Neutrophil extracellular traps kill bacteria," Science, vol. 303, no. 5663, pp. 1532-1535, 2004.

[33] K. Kessenbrock, M. Krumbholz, U. Schönermarck et al., "Netting neutrophils in autoimmune small-vessel vasculitis," Nature Medicine, vol. 15, no. 6, pp. 623-625, 2009. 


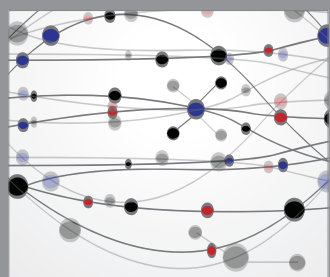

The Scientific World Journal
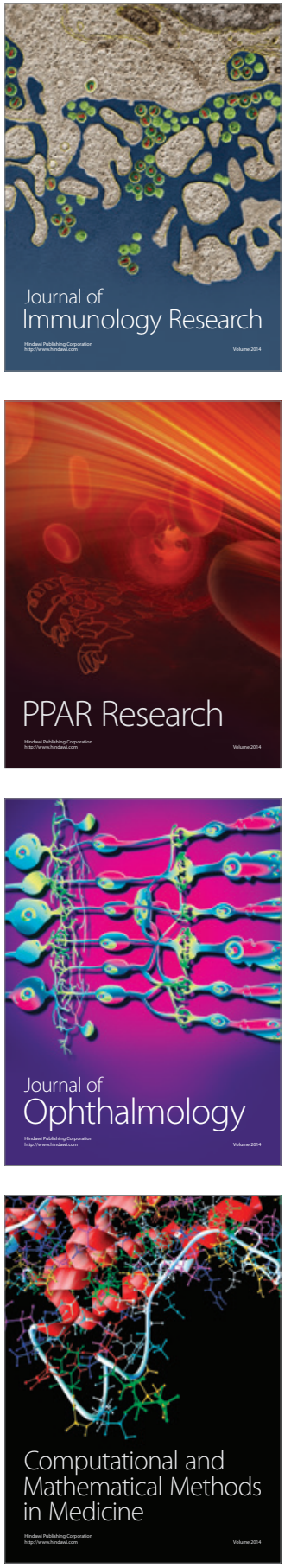

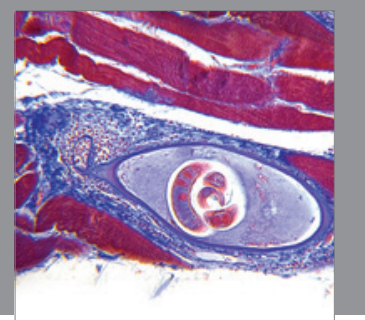

Gastroenterology

Research and Practice
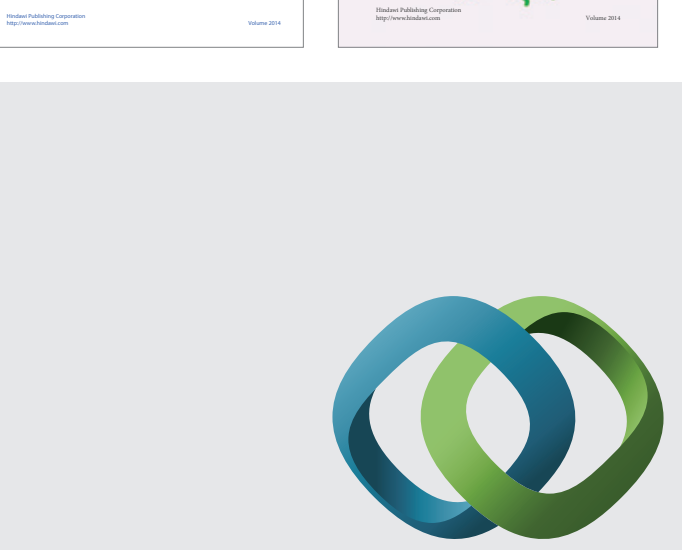

\section{Hindawi}

Submit your manuscripts at

http://www.hindawi.com
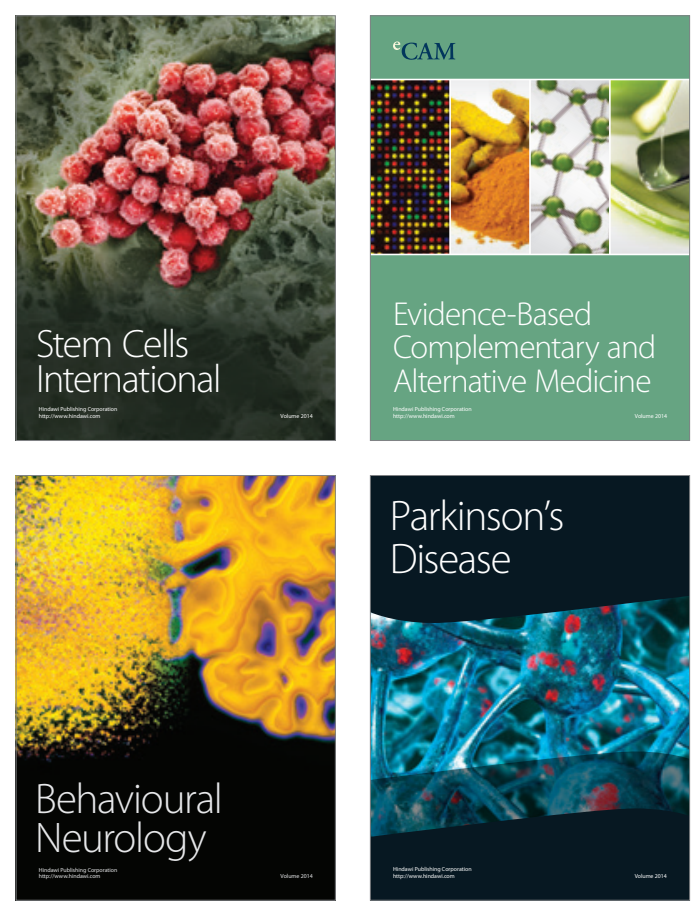

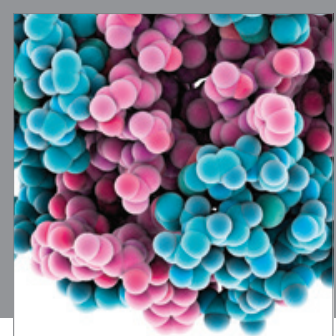

Journal of
Diabetes Research

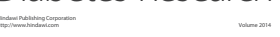

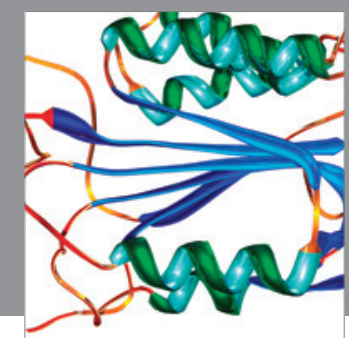

Disease Markers
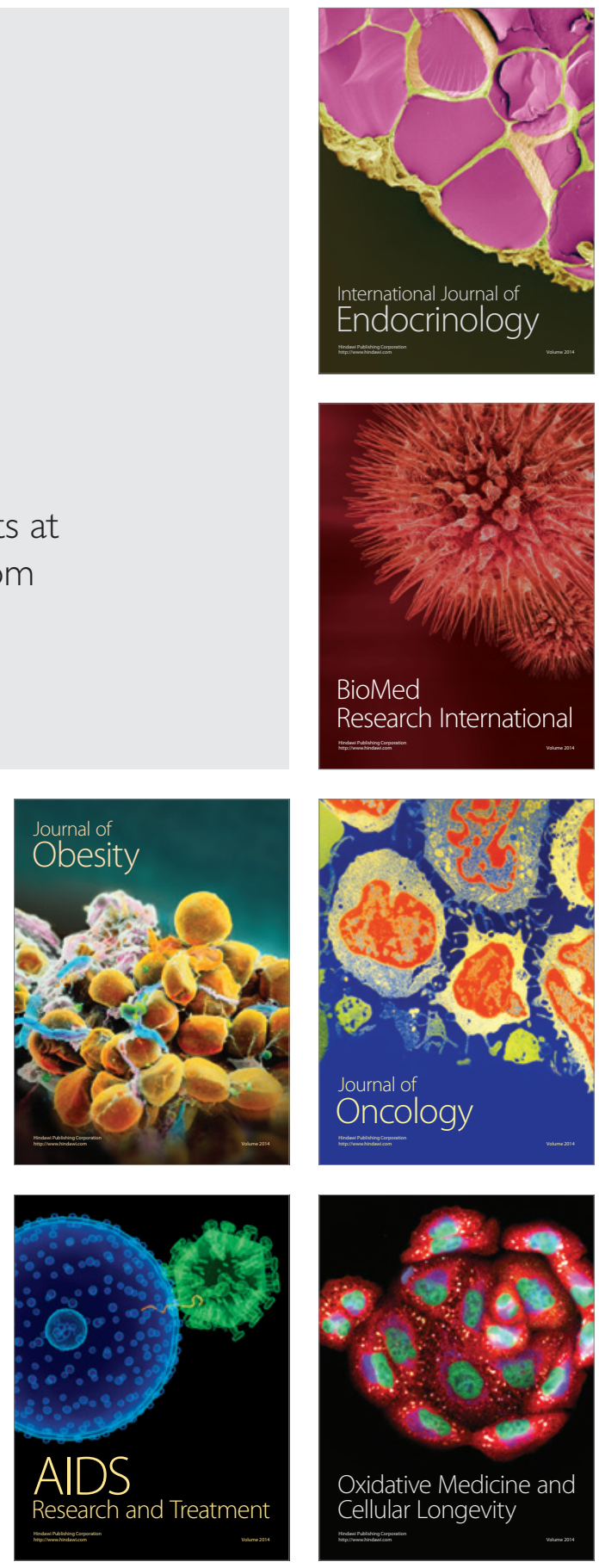